\title{
Targeting Lipid Metabolic Reprogramming as Anticancer Therapeutics
}

\author{
Ji-Young Cha, Ho-Jae Lee \\ Department of Biochemistry, Gachon University College of Medicine, Incheon, Korea
}

\begin{abstract}
Cancer cells rewire their metabolism to satisfy the demands of growth and survival, and this metabolic reprogramming has been recognized as an emerging hallmark of cancer. Lipid metabolism is pivotal in cellular process that converts nutrients into energy, building blocks for membrane biogenesis and the generation of signaling molecules. Accumulating evidence suggests that cancer cells show alterations in different aspects of lipid metabolism. The changes in lipid metabolism of cancer cells can affect numerous cellular processes, including cell growth, proliferation, differentiation, and survival. The potential dependence of cancer cells on the deregulated lipid metabolism suggests that enzymes and regulating factors involved in this process are promising targets for cancer treatment. In this review, we focus on the features associated with the lipid metabolic pathways in cancer, and highlight recent advances on the therapeutic targets of specific lipid metabolic enzymes or regulating factors and target-directed small molecules that can be potentially used as anticancer drugs.
\end{abstract}

(J Cancer Prev 2016;21:209-215)

Key Words: Lipid metabolism, Lipogenesis, Cancer, Therapeutics

\section{INTRODUCTION}

The metabolic properties of cancer cells are different from those of normal cells in that their metabolic machineries are rewired to the demands of growth and survival. This metabolic reprogramming supports the increased production of metabolic intermediates for the synthesis of cellular building blocks and signaling molecules, and has been suggested to a new emerging hallmark of cancer. ${ }^{1}$ The most prominent metabolic alterations in cancer are a high rate of glucose uptake and the use of aerobic glycolysis, called the "Warburg effect", first explored by Otto Warburg et al. ${ }^{2}$ in 1920s. Lipids are energy-rich molecules that serve as major sources of fuel for the cellular metabolic processes. Lipid metabolism, in particular fatty acid (FA) metabolism, is tightly linked to those of glucose and glutamine, since both stimulate FA synthesis by providing substrates, such as acetyl-coenzyme A (acetyl-CoA) and NADPH, as well as cell growth and proliferation. ${ }^{3.4}$ Medes et al. ${ }^{5}$ first demonstrated that FA synthesis occurs at very high rates in cancer tissues, and Ookhtens et al. ${ }^{6}$ showed that cancer cells generate almost all their cellular FAs through de novo biosynthesis. In the last decades, accumulating evidences suggested that the deregulated lipid metabolism has been recognized as one of the most common properties of cancer cells. Like glucose metabolism, lipid metabolism has been associated with cellular proliferation, energy storage, and the generation of signaling molecules, and is believed to be pivotal in the development of cancer. ${ }^{7-10}$ Especially the elevated de novo FA synthesis is one of most prominent aberrations of cancer-associated lipid metabolism. Therefore, targeting lipid metabolic reprogramming might represent a promising strategy in cancer therapy. In this review, we have discussed some of the evidence implicating deregulated lipid biosynthesis in cancer, focusing on key metabolic enzymes in FA synthesis and mobilization. With this focus, we have summarized

Received November 15, 2016, Revised November 25, 2016, Accepted November 26, 2016

Correspondence to: Ho-Jae Lee

Department of Biochemistry, Gachon University College of Medicine, 155 Gaetbeol-ro, Yeonsu-gu, Incheon 21999, Korea

Tel: +82-32-899-6054, Fax: +82-32-899-6039, E-mail: hojlee@gachon.ac.kr, ORCID: Ho-Jae Lee, http://orcid.org/0000-0003-2170-355X

Copyright (C) 2016 Korean Society of Cancer Prevention

(c) This is an Open Access article distributed under the terms of the Creative Commons Attribution Non-Commercial License (http://creativecommons.org/licenses/by-nc/4.0) which permits unrestricted non-commercial use, distribution, and reproduction in any medium, provided the original work is properly cited. 
several anticancer drugs in preclinical trials or under development that are based on targeting altered lipid metabolic pathways in cancer.

\section{MAJOR METABOLIC PATHWAYS OF LIPID BIOSYNTHESIS}

Lipids are hydrophobic molecules which serve as important building blocks of biological membranes. They are used in energy metabolism and storage, and have important roles as signaling molecules in the regulation of numerous biological processes. The majorities of adult mammalian tissues satisfy their lipid requirements through the uptake of lipoproteins and free FAs from the bloodstream. De novo FA and cholesterol biosynthesis are restricted to a subset of tissues, including liver, adipose tissue, and lactating breast. ${ }^{11}$ Most lipids are synthesized from FAs, a diverse class of molecules consisting of carboxylic acid with long aliphatic chains of different lengths and degrees of desaturation. The important metabolic intermediate that provides the substrate for FA and cholesterol biosynthesis is an acetyl-CoA, which is produced through different pathways (Fig. 1). Under aerobic conditions, pyruvate is converted by the enzyme pyruvate dehydrogenase into acetyl-CoA, which enters into tricarboxylic acid (TCA) cycle. Acetyl-CoA is also generated from citrate by cytoplasmic ATP-citrate lyase (ACLY), which cleaves citrate into acetyl-CoA and oxaloacetate. Malonyl-CoA is formed by carboxylation of acetyl-CoA by acetyl-CoA carboxylase (ACC). Acetyl-CoA and malonyl-CoA are then used as the substrates for the production of palmitate, the initial product of FA synthesis, by the multifunctional enzyme fatty acid synthase (FASN). ${ }^{12}$ Palmitate is further elongated by elongase family (elongation of very long chain fatty acid, ELOVL) and desaturated by

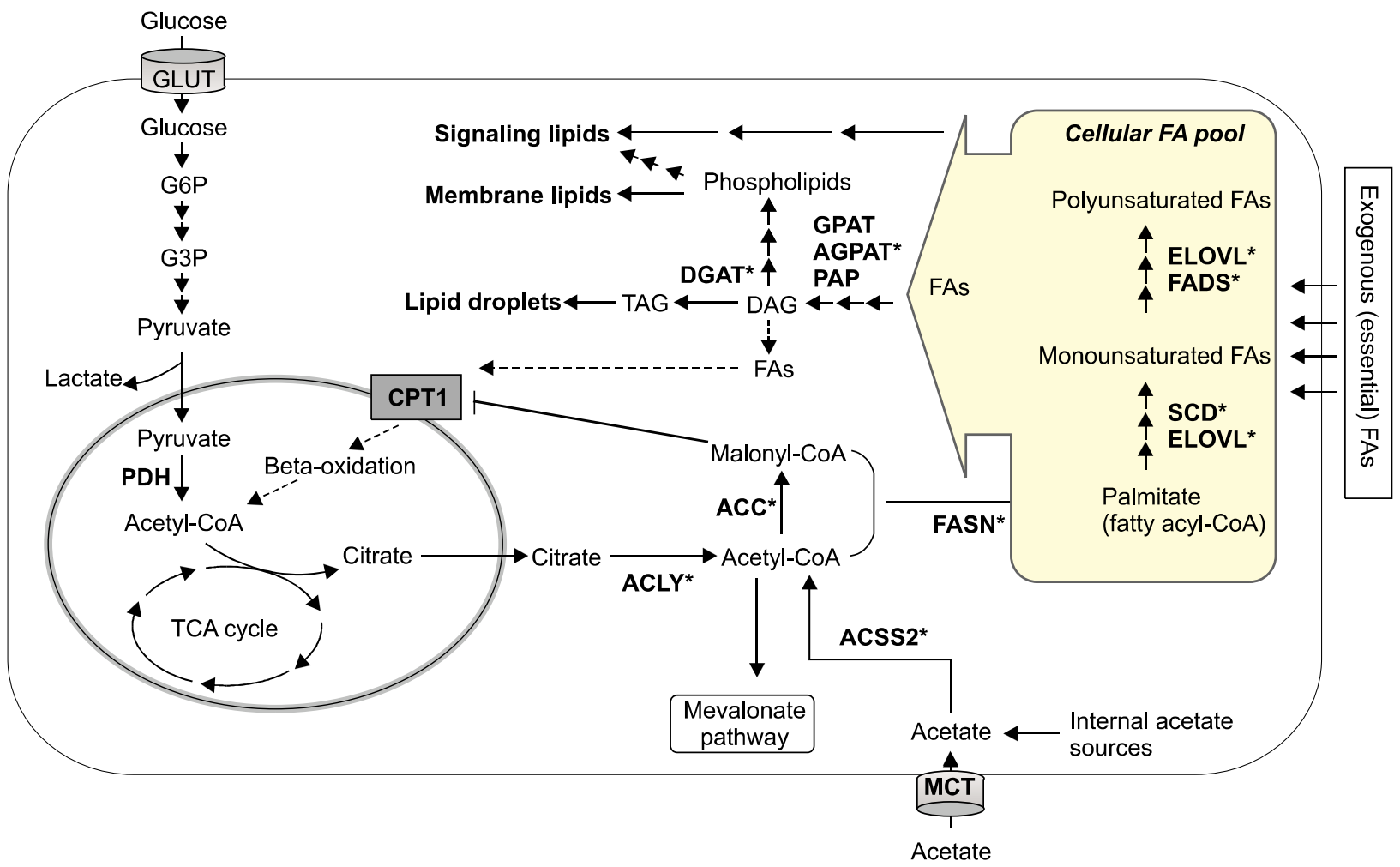

Figure 1. Overview of cellular lipid metabolic pathways. Glucose- or glutamine-derived citrate is first converted to acetyl-coenzyme A (acetyl-CoA) by ATP-citrate lyase (ACLY). Acetyl-CoA can also be synthesized from acetate, which is taken up from the environment or provided by intracellular sources. Acetyl-CoA is carboxylated to malonyl-CoA and condensed by fatty acid (FA) synthase (FASN) in a repeat reaction to generate palmitate. Palmitate is then elongated by FA elongases (elongation of very long chain fatty acids. ELOVLs) and desaturated at the $\triangle 9$ position by stearoyl-CoA desaturases (SCDs). FAs generated through de novo synthesis and from exogenous uptake make up the pool of intracellular FAs. These FAs can be used for energy storage, membrane synthesis, and signaling processes. Activated FAs (fatty acyl-CoA) are converted into FA carnitines by carnitine palmitoyl transferase 1 (CPT1) and broken down by mitochondrial $\beta$-oxidation. Enzymes upregulated or activated in cancer are marked with an asterisk. GLUT, glucose transporter; G6P, glucose-6-phosphate; G3P, glyceraldehyde-3-phosphate; PDH, pyruvate dehydrogenase; TCA, tricarboxylic acid; DGAT, diacylglycerol acyltransferase; GPAT, glycerol-3-phosphate acyltransferase; AGPAT, 1-acylglycerol-3-phosphate-O-acyltransferases; PAP, phosphatidic acid phosphatase; ACC, acetyl-CoA carboxylase; ACSS2, cytoplasmic acetyl-CoA synthetase; FADS, fatty acid desaturase; MCT, monocarboxylate transporter. 
stearoyl-CoA desaturase (SCD) to produce diverse spectrum of saturated and unsaturated FAs. ${ }^{13,14}$

FAs can be used to generate many different types of lipids. FAs are converted into diacylglycerol (DAG) and triacylglycerol (TAG) via the glycerol phosphate pathway, which uses the glycolytic intermediate glycerol-3-phosphate to form the glycerol backbone of these lipids. TAGs are mainly used for energy storage in the form of lipid droplets. Cholesterol, a member of sterol lipids, is a structural component of cellular membranes and plays an important role in membrane dynamics and function. Cholesterol is synthesized from acetyl-CoA via the mevalonate pathway. Acetyl-CoA is first converted into acetoacetyl-CoA and then into mevalonate by 3-hydroxy-3-methly glutaryl (HMG)-CoA synthase and HMG-CoA reductase, respectively. The subsequent conversion of mevalonate to cholesterol involves the coordinated action of numerous enzymes which are part of this pathway.

\section{ABERRANT LIPID METABOLISM IN CANCER}

\section{Fatty acid synthesis}

Cancer cells increase FA biosynthesis to satisfy requirement of lipids for the synthesis of membranes and lipid signaling molecules to trigger cell proliferation leading to malignancy. In addition, cancer cells frequently contain higher lipid accumulation in the form of lipid droplets than that of normal cells. Citrate from TCA cycle in the mitochondria is converted to malonyl-CoA by the action of the enzymes ACLY and ACC (Fig. 1). ACLY is a rate-limiting enzyme and converts mitochondria-derived citrate into acetyl-CoA, which serves as an important precursor for de novo FA synthesis. ACLY is reported to be upregulated in a number of human cancers, including glioblastoma, ovarian cancer, and colorectal cancer, ${ }^{15-17}$ and the inhibition of ACLY reduced cancer cell growth both in vitro and in vivo. ${ }^{18}$ In addition to lipogenesis, ACLY has been found to regulate cellular senescence. The suppression of ACLY in primary cells activates AMP-activated protein kinase (AMPK) which may lead to the activation of tumor suppressor p53, ultimately leading to cellular senescence. ${ }^{19}$

An important step in FA synthesis is the carboxylation of acetyl-CoA in the cytoplasm to form malonyl-CoA. This reaction is catalyzed by ACC, especially ACC $\alpha$, which acts as another rate-limiting enzyme in FA synthesis. Chajès et al. ${ }^{20}$ showed that ACC $\alpha$ is essential to survival of cancer cells. Silencing of ACC $\alpha$ in breast cancer cells results in the induction of apoptosis which may associated with depletion of the cellular pool of FA.
FASN which catalyzes successive condensation reactions in the de novo FA synthesis has been identified as an oncogenic antigen 519 (OA-519) in aggressive breast cancer. ${ }^{21}$ FASN might be the most studied FA metabolic enzyme with respect to cancer. Increased FA synthesis due to the increased levels of FASN has been reported in many types of cancers, including prostate, breast, and other types of cancers, ${ }^{11,22,23}$ and the high expression and activity of FASN correlated with a poor prognosis in cancer patients. ${ }^{24}$ Recently, Wu et al. ${ }^{25}$ showed that cancer cells overexpressing FASN are associated with resistance to DNA-damaging anticancer drugs. Although the exact mechanisms of FASN-mediated drug resistance are not yet clear, they suggest that FASN might contribute to the drug resistance by up-regulating the expression of PARP-1, which in turn activates DNA repair pathways.

SCD catalyzes the critical committed step in the synthesis of monounsaturated FAs from saturated FAs. Monounsaturated FAs are important components in membrane phospholipids, as well as the precursors of DAG, TAG, and cholesterol esters. Changes in physical properties of FA and lipid composition can affect membrane fluidity, signaling, and cellular activities. Two isoforms of SCD, SCD-1 and SCD-5, have been identified in human. SCD-1 is highly expressed in oncogene-transformed lung fibroblasts and cancer cells, and its importance for cancer lipid metabolism has been increasingly recognized. ${ }^{26}$

In addition to key enzymes involved in FA synthesis, transcriptional regulators responsible for their expressions are also deregulated in cancers. The important transcriptional activators for FA synthesis are sterol regulatory element-binding proteins (SREBPS). ${ }^{27}$ SREBPs, a family of three basic helix-loop-helix-leucine zipper transcription factors, regulate lipogenic processes by binding SREs and E-box sequences in the promoters of their target genes including ACLY, ACC $\alpha$, FASN, and SCD- $1 .{ }^{28}$ The aberrant activation of SREBPs and induction of their target genes have also been involved in cancer development. ${ }^{29,30}$

Acyl-CoA synthetase (ACS), also known as FA-CoA ligase, catalyzes the conversion of long-chain FA to acyl-CoA. This reaction is a critical step in some lipid metabolic pathways, including phospholipid synthesis, lipid modification of cellular proteins, and FA oxidation. Mammals have five ACS isoforms and also have FA transport proteins with ACS activity. Different types of ACS isoforms, ACSL4 and ACSL5, have been found to be upregulated in colon cancer and glioblastoma, respectively. ${ }^{31,32}$ These evidences suggest that ACSs are significantly involved in cancer cell proliferation and survival. 


\section{Fatty acid oxidation}

Unlike FA synthesis, FA oxidation, also known as $\beta$-oxidation, in cancer cells remains largely unknown. However, several lines of evidence demonstrated that the degree of FA oxidation contributes cancer metabolic transformation. ${ }^{30}$ For FA oxidation, FA-CoA, the activated FA, has to be transported from cytosol across the mitochondrial membrane after it is converted to FA carnitines by carnitine palmitoyltransferase 1 (CPT1). Zaugg et $\mathrm{al}^{33}$ showed that a CPT isoform CPT1C is overexpressed in many human cancer cells, and the suppression of CPT1C reduces cell growth in vitro, as well as new tumor formation in vivo.

\section{Fatty acid storage and mobilization}

The active FA pool available for cancer cell growth and proliferation can be changed by diverting FAs to storage or by blocking FA release from storage. Most cells store FAs in TAGs in the cytosolic lipid droplets. Although increased numbers of lipid droplets have been reported in many cancer cells, ${ }^{34}$ the exact role of lipid droplets in cancer is still unclear. The synthesis of TAG is initiated with successive esterification of the hydroxyl groups with various FAs on the glycerol-3-phosphate backbone. These acylation processes are sequentially catalyzed by glycerol-3-ph- osphate acyltransferases (GPATs), 1-acylglycerol-3-phosphate-Oacyltransferases (AGPATs), and diacylglycerol acyltransferases. ${ }^{35}$ Agarwal $^{36}$ showed that different isoforms of AGPATs are upregulated in a variety of cancers.

Storage lipids provide a reservoir of FAs that can be mobilized for energy generation of cancer cells through the action of lipases, such as hormone-sensitive lipase, adipose triglyceride lipase, and monoacylglycerol lipase (MAGL). The FAs derived from lipolysis can also serve as precursors for important signaling intermediates of lipids. Nomura et $\mathrm{al}^{37}$ showed that the expression of MAGL is highly increased in aggressive human cancer cells and primary tumors, in which it regulates a FA network enriched in oncogenic signaling lipids that promotes migration, invasion, survival, and tumor growth.

\section{ANTICANCER AGENTS TARGETING LIPID METABOLIC REPROGRAMMING}

Since FAs are essential for cancer cell proliferation and survival, limiting their lipid pool availability might provide a promising therapeutic strategy. Some of the recently developed small-molecule agents targeting the lipid metabolic enzymes and upstream signaling transcription factors that linked to cancer

Table 1. Small-molecule inhibitors targeting lipid metabolic pathways for cancer treatment

\begin{tabular}{|c|c|c|c|}
\hline Target & Compound & Mechanism of action & Effect \\
\hline \multirow[t]{5}{*}{$\begin{array}{l}\text { Fatty acid synthase } \\
\text { (FASN) }\end{array}$} & $\begin{array}{l}\text { Cerulenin and its } \\
\text { derivative } \mathrm{C} 75, \mathrm{C} 93\end{array}$ & Inhibition of $\beta$-ketoacyl synthase activity & $\begin{array}{l}\text { Inhibition of proliferation, induction of } \\
\text { apoptosis, reduction of tumor growth }\end{array}$ \\
\hline & Orlistat & Inhibition of thioesterase activity & $\begin{array}{l}\text { Induction of apoptosis, reduction of } \\
\text { tumor growth }\end{array}$ \\
\hline & GSK2194069 & Inhibition of $\beta$-ketoacyl reductase domain & Reduction of tumor growth \\
\hline & Triclosan & $\begin{array}{l}\text { Inhibition of enoyl-acyl carrier protein } \\
\text { reductase domain reductase domain }\end{array}$ & $\begin{array}{l}\text { Induction of apoptosis, induction of } \\
\text { senescence }\end{array}$ \\
\hline & Fasnall & Binding of co-factor sites of FASN & Inhibition of tumor growth \\
\hline $\begin{array}{l}\text { ATP-citrate lyase } \\
\text { (ACLY) }\end{array}$ & SB-204990 & Inhibition of ACLY & Inhibition of tumor growth \\
\hline $\begin{array}{l}\text { Acetyl-CoA carboxylase } \\
\text { (ACC) }\end{array}$ & $\begin{array}{l}\text { TOPA } \\
\text { Soraphen A }\end{array}$ & Inhibition of $\mathrm{ACCs}$ & Induction of apoptosis \\
\hline $\begin{array}{l}\text { Stearoyl-CoA desaturase } \\
\text { (SCD) }\end{array}$ & $\begin{array}{l}\text { BZ36 } \\
\text { A939572 }\end{array}$ & Inhibition of SCD & $\begin{array}{l}\text { Inhibition of tumor growth } \\
\text { Reduction of tumor growth }\end{array}$ \\
\hline $\begin{array}{l}\text { Sterol regulatory } \\
\text { element-binding } \\
\text { protein (SREBP) }\end{array}$ & $\begin{array}{l}\text { Fatostatin } \\
\text { Betulin }\end{array}$ & Inhibition of SREBP processing & Inhibition of proliferation \\
\hline $\begin{array}{l}\text { Acetyl-CoA synthase } \\
\text { (ACS) }\end{array}$ & Triacscin C & Inhibition of ACSs & $\begin{array}{l}\text { Induction of apoptosis, inhibition of } \\
\text { tumor growth }\end{array}$ \\
\hline $\begin{array}{l}\text { Carnitine palmitoyl } \\
\text { transferase } 1 \text { (CPT1) }\end{array}$ & $\begin{array}{l}\text { Etomoxir } \\
\text { Perhexiline }\end{array}$ & Inhibition of CPT1 & Inhibition of proliferation \\
\hline $\begin{array}{l}\text { Acylglycerolphosphate } \\
\text { acyltransferase (AGPAT) }\end{array}$ & CT-32501 & Inhibition of AGPAT2 & Inhibition of tumor growth \\
\hline $\begin{array}{l}\text { Monoacylglycerol lipase } \\
\text { (MAGL) }\end{array}$ & JZL184 & Inhibition of MAGL & Induction of apoptosis \\
\hline
\end{tabular}


treatment are shown in Table 1. Inhibitors directly targeting lipogenic enzymes, such as FASN, ACLY, and ACC, have been the subject of intense studies, and their efficacy as anticancer agents has been proven in various models of cancers.

\section{Fatty acid synthesis}

Early studies demonstrated that FASN is a particularly attractive therapeutic target because most cancer cells depend on FASN-mediated de novo FA synthesis, whereas most normal cells prefer exogenous sources. Several chemical inhibitors of FASN have been developed and evaluated in preclinical models. An antifungal antibiotic, cerulenin, is the first known FASN inhibitor. It is a potent non-competitive irreversible inhibitor of FASN by binding to the active site of $\beta$-ketoacyl reductase domain of FASN. ${ }^{38}$ Cerulenin significantly reduces cancer cell FA synthesis and induces the regression of established tumors. ${ }^{39}$ Similarly, the semi-synthetic analogue of cerulenin $\mathrm{C} 75$ shows anti-tumorigenic activity in xenograft models of human cancers. ${ }^{9}$ An anti-obesity drug orlistat is also reported to inhibit FASN and to suppress growth of xenografts in mice. ${ }^{40}$ However, orlistat has a poor solubility and oral bioavailability, thereby its clinical application for systemic use in cancer treatment is limited. ${ }^{41}$ In addition, an antimicrobial agent triclosan ${ }^{42}$ and GSK2194069 $9^{43}$ have been shown to have a potent inhibitory activity in FASN. Recently, Alwarawrah et al. $^{44}$ showed that Fasnall, a novel thiophenopyrimidine-based FASN inhibitor, exerts potent antitumor activity against various breast cancer cell lines and is well tolerated in mice. Moreover, Fasnall reduces tumor volume and increases survival in a mouse breast cancer model. Until now, the majority of studies targeting lipid metabolic reprogramming have focused on FASN, however, it seems that the adverse side effects of FASN-targeting agents have precluded their clinical development.

The increased activity and expression of ACLY in cancer cells also suggest that the inhibition of ACLY may be a promising approach for cancer therapy. Several inhibitors of ACLY have been primarily evaluated for their ability to block FA or cholesterol synthesis. Pharmacological inhibition of ACLY by SB-204990 efficiently blocks cancer cell growth and cell cycle entry, and suppresses tumor growth in xenografts. ${ }^{45}$ Soraphen A, a natural macrocyclic polyketide which have a potent ACC inhibitory activity, induces apoptosis of prostate cancer cells. ${ }^{46}$ However, a contradictory result has been observed with ACC inhibition by 5-(tetradecyloxy)-2-furoic acid (TOFA) in breast cancer cells. ${ }^{47}$ The inhibition of SCD1 by BZ36 impairs FA synthesis and restrains tumor growth in preclinical cancer models ${ }^{48}$ without affecting overall body weight. Additionally, A939572, a SCD1 inhibitor, delays tumor growth in a human gastric cancer xenograft model. ${ }^{49}$

Targeting master transcriptional factors may be an efficient strategy to inhibit and suppress cancer growth. Fatostatin, a chemical SREBP inhibitor, has been found to suppress prostate cancer cell proliferation and reduce tumor growth. ${ }^{50}$ In addition, the natural compound betulin, which inhibits SREBP activation, has been shown to have broad spectrum of anticancer activity in vitro and in vivo. ${ }^{51}$ Inhibition of ACS by Triacscin C significantly induces apoptosis in p53-defective human cancer cells and suppresses growth of xenograft tumors. ${ }^{52}$

\section{Fatty acid oxidation and mobilization}

In some cancer types, increased FA oxidation may limit FA availability. On the other hand, the use of CPT1 inhibitor, etomoxir, has been found to be beneficial in FA oxidation-dependent tumors, notably in prostate cancer. ${ }^{53}$ Recently, perhexiline, an antianginal drug that inhibits CPT, has been shown to be highly effective in killing chronic lymphocytic leukemia cells in stromal microenvironment at clinically achievable concentrations and significantly prolonged animal survival. ${ }^{54}$ Takeuchi and Reue ${ }^{55}$ showed that elevated expression of AGPAT2 is associated with poor prognosis of ovarian cancer cells, and an AGPAT2 inhibitor CT-30501 has antitumor activity in xenograft model. MAGL hydrolyzes intracellular TAGs stored in adipocytes and other cells to FAs and glycerol. Pharmacological inhibition of MAGL by JZL184 decreases free FA levels and impairs cancer pathogenicity of melanoma and ovarian cancer cells. ${ }^{37}$ Interestingly, the impairments in MAGL-dependent tumor growth are rescued by a high-fat diet, indicating that exogenous sources of FAs can contribute to malignancy in cancers lacking MAGL activity.

\section{CONCLUSION}

Advancements over the past decades have shown that cancer cells reprogram their lipid metabolism to facilitate growth and proliferation. Therefore, the signaling pathways controlling the aberrant lipid metabolism in cancer are attractive targets for cancer therapy. The elevated expression and activity of FASN in most types of cancer cells and the important role of FASN in maintaining cancer phenotype, cell growth and survival indicate that FASN is considered as a promising therapeutic target for cancer treatment. Numerous efforts show that the pharmacological inhibition of FASN is effective in vitro and in xenograft models. Although the results of these studies are encouraging, the problem of predictable side effects of targeting FASN still 
remains. In addition, cancers are diverse in type and lipid metabolism is complex, with many different feedback mechanisms and points of regulation. Therefore, further investigation on the regulation of signaling pathways of lipid metabolism in cancer cells may provide rational targets for therapy, and defining the role of diverse lipid signaling molecules will offer novel therapeutic opportunities for the development of anticancer drugs.

\section{ACKNOWLEDGMENTS}

This work was supported by the Basic Science Research Program through the National Research Foundation of Korea (NRF) funded by the Ministry of Education, Science and Technology (NRF-2016R1A2B4015866) to J-Y Cha and by the Gachon University Gil Medical Center Research Fund (FRD2013-17) to H-J Lee.

\section{CONFLICTS OF INTEREST}

No potential conflicts of interest were disclosed.

\section{REFERENCES}

1. Hanahan D, Weinberg RA. Hallmarks of cancer: the next generation. Cell 2011;144:646-74.

2. Warburg O, Wind F, Negelein E. The metabolism of tumors in the body. J Gen Physiol 1927;8:519-30.

3. DeBerardinis RJ, Lum JJ, Hatzivassiliou G, Thompson CB. The biology of cancer: metabolic reprogramming fuels cell growth and proliferation. Cell Metab 2008;7:11-20.

4. Kim MH, Kim H. Oncogenes and tumor suppressors regulate glutamine metabolism in cancer cells. J Cancer Prev 2013;18:221-6.

5. Medes G, Thomas A, Weinhouse S. Metabolism of neoplastic tissue. IV. A study of lipid synthesis in neoplastic tissue slices in vitro. Cancer Res 1953;13:27-9.

6. Ookhtens M, Kannan R, Lyon I, Baker N. Liver and adipose tissue contributions to newly formed fatty acids in an ascites tumor. Am J Physiol 1984;247:R146-53.

7. Baenke F, Peck B, Miess H, Schulze A. Hooked on fat: the role of lipid synthesis in cancer metabolism and tumour development. Dis Model Mech 2013;6:1353-63.

8. Menendez JA, Lupu R. Oncogenic properties of the endogenous fatty acid metabolism: molecular pathology of fatty acid synthase in cancer cells. Curr Opin Clin Nutr Metab Care 2006;9:346-57.

9. Röhrig F, Schulze A. The multifaceted roles of fatty acid synthesis in cancer. Nat Rev Cancer 2016;16:732-49.

10. Santos CR, Schulze A. Lipid metabolism in cancer. FEBS J 2012;279:2610-23.

11. Menendez JA, Lupu R. Fatty acid synthase and the lipogenic phenotype in cancer pathogenesis. Nat Rev Cancer 2007;7:763-77.

12. Maier T, Leibundgut M, Ban N. The crystal structure of a mammalian fatty acid synthase. Science 2008;321:1315-22.

13. Jakobsson A, Westerberg R, Jacobsson A. Fatty acid elongases in mammals: their regulation and roles in metabolism. Prog Lipid
Res 2006:45:237-49

14. Paton CM, Ntambi JM. Biochemical and physiological function of stearoyl-CoA desaturase. Am J Physiol Endocrinol Metab 2009;297:E28-37.

15. Beckner ME, Fellows-Mayle W, Zhang Z, Agostino NR, Kant JA, Day BW, et al. Identification of ATP citrate lyase as a positive regulator of glycolytic function in glioblastomas. Int J Cancer 2010;126:2282-95.

16. Wang Y, Wang Y, Shen L, Pang Y, Qiao Z, Liu P. Prognostic and therapeutic implications of increased ATP citrate lyase expression in human epithelial ovarian cancer. Oncol Rep 2012;27:1156-62.

17. Zhou Y, Bollu LR, Tozzi F, Ye X, Bhattacharya R, Gao G, et al. ATP citrate lyase mediates resistance of colorectal cancer cells to SN38. Mol Cancer Ther 2013;12:2782-91.

18. Zaidi N, Swinnen JV, Smans K. ATP-citrate lyase: a key player in cancer metabolism. Cancer Res 2012;72:3709-14.

19. Lee JH, Jang H, Lee SM, Lee JE, Choi J, Kim TW, et al. ATP-citrate lyase regulates cellular senescence via an AMPK- and p53-dependent pathway. FEBS J 2015;282:361-71.

20. Chajès V, Cambot M, Moreau K, Lenoir GM, Joulin V. Acetyl-CoA carboxylase alpha is essential to breast cancer cell survival. Cancer Res 2006;66:5287-94.

21. Kuhajda FP, Jenner K, Wood FD, Hennigar RA, Jacobs LB, Dick JD, et al. Fatty acid synthesis: a potential selective target for antineoplastic therapy. Proc Natl Acad Sci U S A 1994:91:6379-83.

22. Swinnen JV, Vanderhoydonc F, Elgamal AA, Eelen M, Vercaeren I, Joniau S, et al. Selective activation of the fatty acid synthesis pathway in human prostate cancer. Int J Cancer 2000;88:176-9.

23. Yoon S, Lee MY, Park SW, Moon JS, Koh YK, Ahn YH, et al. Up-regulation of acetyl-CoA carboxylase alpha and fatty acid synthase by human epidermal growth factor receptor 2 at the translational level in breast cancer cells. J Biol Chem 2007;282:26122-31.

24. Kuhajda FP. Fatty acid synthase and cancer: new application of an old pathway. Cancer Res 2006;66:5977-80.

25. Wu X, Qin L, Fako V, Zhang JT. Molecular mechanisms of fatty acid synthase (FASN)-mediated resistance to anti-cancer treatments. Adv Biol Regul 2014;54:214-21.

26. Igal RA. Stearoyl-CoA desaturase-1: a novel key player in the mechanisms of cell proliferation, programmed cell death and transformation to cancer. Carcinogenesis 2010;31:1509-15.

27. Horton JD, Goldstein JL, Brown MS. SREBPs: activators of the complete program of cholesterol and fatty acid synthesis in the liver. J Clin Invest 2002;109:1125-31.

28. Sun Y, He W, Luo M, Zhou Y, Chang G, Ren W, et al. SREBP1 regulates tumorigenesis and prognosis of pancreatic cancer through targeting lipid metabolism. Tumour Biol 2015;36:4133-41.

29. Shao W, Espenshade PJ. Expanding roles for SREBP in metabolism. Cell Metab 2012;16:414-9.

30. Swinnen JV, Brusselmans K, Verhoeven G. Increased lipogenesis in cancer cells: new players, novel targets. Curr Opin Clin Nutr Metab Care 2006;9:358-65.

31. Cao Y, Pearman AT, Zimmerman GA, McIntyre TM, Prescott SM. Intracellular unesterified arachidonic acid signals apoptosis. Proc Natl Acad Sci U S A 2000;97:11280-5.

32. Yamashita Y, Kumabe T, Cho YY, Watanabe M, Kawagishi J, Yoshimoto T, et al. Fatty acid induced glioma cell growth is mediated by the acyl-CoA synthetase 5 gene located on chromosome 10q25.1-q25.2, a region frequently deleted in malignant gliomas. 
Oncogene 2000;19:5919-25.

33. Zaugg K, Yao Y, Reilly PT, Kannan K, Kiarash R, Mason J, et al. Carnitine palmitoyltransferase $1 \mathrm{C}$ promotes cell survival and tumor growth under conditions of metabolic stress. Genes Dev 2011;25:1041-51.

34. Farese RV Jr, Walther TC. Lipid droplets finally get a little R-E-S-P-E-C-T. Cell 2009;139:855-60.

35. Coleman RA, Lee DP. Enzymes of triacylglycerol synthesis and their regulation. Prog Lipid Res 2004:43:134-76.

36. Agarwal AK. Lysophospholipid acyltransferases: 1-acylglycerol-3-phosphate O-acyltransferases. From discovery to disease. Curr Opin Lipidol 2012;23:290-302.

37. Nomura DK, Long JZ, Niessen S, Hoover HS, Ng SW, Cravatt BE. Monoacylglycerol lipase regulates a fatty acid network that promotes cancer pathogenesis. Cell 2010;140:49-61.

38. Goldberg I, Walker JR, Bloch K. Inhibition of lipid synthesis in Escherichia coli cells by the antibiotic cerulenin. Antimicrob Agents Chemother 1973;3:549-54.

39. Pizer ES, Wood FD, Heine HS, Romantsev FE, Pasternack GR, Kuhajda FP. Inhibition of fatty acid synthesis delays disease progression in a xenograft model of ovarian cancer. Cancer Res 1996:56:1189-93.

40. Kridel SJ, Axelrod F, Rozenkrantz N, Smith JW. Orlistat is a novel inhibitor of fatty acid synthase with antitumor activity. Cancer Res 2004:64:2070-5.

41. Liu H, Liu JY, Wu X, Zhang JT. Biochemistry, molecular biology, and pharmacology of fatty acid synthase, an emerging therapeutic target and diagnosis/prognosis marker. Int J Biochem Mol Biol 2010;1:69-89.

42. Sadowski MC, Pouwer RH, Gunter JH, Lubik AA, Quinn RJ, Nelson CC. The fatty acid synthase inhibitor triclosan: repurposing an anti-microbial agent for targeting prostate cancer. Oncotarget 2014:5:9362-81.

43. Hardwicke MA, Rendina AR, Williams SP, Moore ML, Wang L, Krueger JA, et al. A human fatty acid synthase inhibitor binds $\beta$-ketoacyl reductase in the keto-substrate site. Nat Chem Biol 2014:10:774-9.

44. Alwarawrah Y, Hughes P, Loiselle D, Carlson DA, Darr DB, Jordan JL, et al. Fasnall, a selective fasn inhibitor, shows potent anti-tumor activity in the MMTV-Neu model of HER2(+) breast cancer. Cell Chem Biol 2016;23:678-88.
45. Hatzivassiliou G, Zhao F, Bauer DE, Andreadis C, Shaw AN, Dhanak D, et al. ATP citrate lyase inhibition can suppress tumor cell growth. Cancer Cell 2005;8:311-21.

46. Beckers A, Organe S, Timmermans L, Scheys K, Peeters A, Brusselmans K, et al. Chemical inhibition of acetyl-CoA carboxylase induces growth arrest and cytotoxicity selectively in cancer cells. Cancer Res 2007;67:8180-7.

47. Pizer ES, Thupari J, Han WF, Pinn ML, Chrest FJ, Frehywot GL, et al. Malonyl-coenzyme-A is a potential mediator of cytotoxicity induced by fatty-acid synthase inhibition in human breast cancer cells and xenografts. Cancer Res 2000;60:213-8.

48. Fritz V, Benfodda $Z$, Rodier $G$, Henriquet $C$, Iborra $F$, Avancès $C$, et al. Abrogation of de novo lipogenesis by stearoyl-CoA desaturase 1 inhibition interferes with oncogenic signaling and blocks prostate cancer progression in mice. Mol Cancer Ther 2010;9:1740-54.

49. Roongta UV, Pabalan JG, Wang X, Ryseck RP, Fargnoli J, Henley BJ, et al. Cancer cell dependence on unsaturated fatty acids implicates stearoyl-CoA desaturase as a target for cancer therapy. Mol Cancer Res 2011;9:1551-61.

50. Li X, Chen YT, Hu P, Huang WC. Fatostatin displays high antitumor activity in prostate cancer by blocking SREBP-regulated metabolic pathways and androgen receptor signaling. Mol Cancer Ther 2014:13:855-66.

51. Król SK, Kiełbus M, Rivero-Müller A, Stepulak A. Comprehensive review on betulin as a potent anticancer agent. Biomed Res Int 2015;2015:584189.

52. Mashima T, Oh-hara T, Sato S, Mochizuki M, Sugimoto $Y$, Yamazaki K, et al. p53-defective tumors with a functional apoptosome-mediated pathway: a new therapeutic target. J Natl Cancer Inst 2005;97:765-77.

53. Schlaepfer IR, Rider L, Rodrigues LU, Gijón MA, Pac CT, Romero $\mathrm{L}$, et al. Lipid catabolism via CPT1 as a therapeutic target for prostate cancer. Mol Cancer Ther 2014;13:2361-71.

54. Liu PP, Liu J, Jiang WQ, Carew JS, Ogasawara MA, Pelicano H, et al. Elimination of chronic lymphocytic leukemia cells in stromal microenvironment by targeting CPT with an antiangina drug perhexiline. Oncogene 2016;35:5663-73.

55. Takeuchi K, Reue K. Biochemistry, physiology, and genetics of GPAT, AGPAT, and lipin enzymes in triglyceride synthesis. Am J Physiol Endocrinol Metab 2009:296:E1195-209. 